zeigt, dass die Grenze unseres Vermögens durch die Schwere der Verletzung gegeben wird. Fall 4 lehrt, dass das bisherige Verfahren unentbehrlich bleibt.

Leider ist die Gruppe a ausserordentlich viel seltener als b und $c$, wenigstens bei unseren Arbeitern.

B. Die zweite Mittheilung aus Prof. Haab's Klinik ist von A. Hürzeler ${ }^{3}$ ).

In Zürich ergaben in vier Jahren vor der Magnetanwendung (1877-1880) 24 Fälle von Eisensplittern im Glaskörper 24 Verluste, also $100 \%$; in vier Jahren mit Magnetoperation und Antisepsis (1883-1886) 35 Fälle nur noch 24 Verluste, d. h. blos $68 \%$.

Die Magnetsondirung hält er für schädlich, wegen der allseitigen Durchwïhlung des Glaskörpers. Aber wer nöthigt den Wundarzt, so regelwidrig vorzugehen? II, Fall $42-47$, habe ich sechs Fälle beschrieben, wo die (nach der Art der Verletzung) gebotene Magnetsondirung völlig erfolglos blieb und doch ganz gute, sogar volle Sehschärfe zurückliess: und darauf folgen noch etliche Fälle der Art, wo das Auge nicht die geringste Schädigung von der vorsichtigen Sondirung erlitt, wenn auch die von dem verletzten Arbeiter zugestandene Sehkraft, im Widerspruch mit dem objectiven Befund, nur mässig war.

Die Statistik, die Verfasser von meinen Fällen macht, ist unrichtig. Die vier vorzüglichen und über viele Jahre beobachteten Fälle beziehen sich nicht auf „65 Splitterextractionen aus dem Glaskörper", sondern auf 41; wenn man die Fälle abzieht, wo der Splitter aus mechanischen Gründen überhaupt nicht ausziehbar war, auf 33; und wenn, wie billig, bei Beurtheilung des Seherfolges, diejenigen Fälle abgezogen werden, die schon mit Glaskörpervereiterung kamen, auf 22 . Und darunter sind noch dazu meine ersten Versuche, sogar mein erster Operationsfall aus dem Jahre 1875, wo ich meinen Elektromagneten noch nicht besass. Also auf 22 oder 21 Fälle, mit denen noch etwas zu machen war, vier gute Seherfolge und drei mittelmässige, dazı sechsmal Erhaltung der Form des Augapfels. Zu Zürich wurde freilich unter 18 Fällen nur einmal eine leidliche Sehschärfe $(1 / 7)$ erzielt.

Ich selber habe absichtlich bisher noch niemals eine Statistik

\section{Ueber die Entfernung von Eisensplittern aus der Netzhaut.}

\section{Von Prof. Dr. J. Hirschberg in Berlin.}

(Schluss aus No. 23.)

Nachdem ich das bisherige Verfahren gegen unbegründete Vorwürfe vertheidigt und seine Leistungsfähigkeit durch einige neue Beispiele klargelegt, gehe ich nunmehr dazu über, die neue Behandlungsweise genauer $\mathrm{zu}$ betrachten.

A. Auf dem Heidelberger Ophthalmologen-Congress von 1892 machte Prof. Ha ab eine interessante Mittheilung ${ }^{1}$ ):

Fall 1. Eine 32jährige Frau hatte seit drei Wochen in der hintersten Schicht der Linse einen Eisensplitter, der etwa $1 \mathrm{~mm}$ weit in den Glaskörper hervorragte. Haab legte das verletzte Auge dicht an die scharfe Ecke des $7 \mathrm{~cm}$ dicken Eisenkernes eines grossen Ruhmkorffschen ${ }^{2}$ ) Apparates, der seinen Strom von einer Batterie von sechs grossen Accumulatoren bekam. Im $\mathrm{Nu}$ war der Splitter in der vorderen Augenkammer und konnte leicht entfernt werden. (Später wurde die getrubte Linse durch Lanzenschnitt entleert. Sehschärfe $=1 / 4$ ).

Fall 2. Einem 22jährigen war ein Eisensplitter durch die Linse in den Glaskorper gedrungen. Zwei Tage später wurde das Auge unter Hin- und Herbewegung 3-4 Minuten in möglichster Nähe der Ecke des Eisenkernes gehalten; der Splitter erschien in der vorderen Kapselwunde und konnte leicht entfernt werden. (Später Discission, Sehschärfe $=1 / 2$ ).

Fall 3. Desgleichen bei einem 54jährigen, wo der Splitter vor fünf Tagen durch die Linse in den Glaskörper gedrungen und dort einen Abscess bewirkt hatte; das A uge musste aber 14 Tage später doch entfernt werden.

Fall 4. Einer 35jährigen war vor $1 \frac{1}{2}$ Monaten ein Splitter durch Hornhaut und Linse eingedrungen und sass unten in der Netzhaut fest. Der Versuch mit dem grossen Magnet war erfolglos, Skleralschnitt und Eingehen mit dem kleinen Magnet förderte "nach vieler Mühe und lanoem Suchen" den Splitter heraus; es erfolgte zuerst schöne Heilung. (Aber nachträglich Netzhautablösung und Erblindung des Auges.)

$\mathrm{Ha}$ a b ist der Ansicht, dass der Splitter hierbei aus dem Glaskörper den $W$ undeanal zurückverfolgt (a). Darum dürfte so die Ausziehung nicht gelingen, wenn der Splitter hinten zurückgeprallt ist und lose im Glaskörper liegt (b). Festsitzende und eingekapselte Splitter können so nicht entfernt werden (c).

Fall 1 ist nach meiner Ansicht leicht und sicher nach dem bisherigen Verfahren zu operiren. Fall 2 ist überraschend, obwohl auch dieser nach dem bisherigen operirt werden konnte: wie ich an einem ganz gleichartigen alsbald nachweisen werde. Fall 3

1) Bericht S. 162 .

2) "Rumkorff" sollte der Heidelberger Bericht nicht schreiben. der Magnetoperationen mit Procenten des Verlustes und der Erfolge veröffentlicht, weil ich dazu noch mehr Fälle sammeln möchte. Die Operationen verschiedener Wundärzte zusammenzustellen, ist gerade auf diesem Gebiete unthunlich. Das hiesse, incommensurable Grössen zusammenfügen. Der eine holt in 17 von 28 Fällen den Splitter mit dem Elektromagneten, der andere in allen von ihm operirten Fällen niemals. Der eine hat unter den 28 Fällen zehnmal einen grösseren oder geringeren Bruchtheil von Sehschärfe; der andere in zehn Fällen niemals, ein dritter in sieben Fällen niemals Sehkraft erhalten.

Zum Schluss folgen die von Haab mit dem grossen Magnet operirten Fälle. (Fall 1-4, vgl. A).

Fall 5. Ein 20 jähriger kummt sofort nach der Verletzung des linken Auges. Wunde von $4 \mathrm{~mm}$, am unteren Rand der Hornhaut und in der angrenzenden Lederhaut. Splitter im Glaskörper sichtbar. Am folgenden Tage wird im Polytechnicum der Splitter mittels des grossen Magneten ausgezogen $(17 \times 21 / 2 \mathrm{~mm} ; 315 \mathrm{mg})$, dabei etwas Iris eingeklemmt und diese, nach der Rïckkehr in's Spital, abgetragen. Nach einem halben Jahre Finger auf $1,5 \mathrm{~m}$.

Fall 6. Einem 15jührigen drang ein kleiner Splitter durch den lateralen Rand der Hornhaut, Linse, Glaskörper ein und steckte aussenoben vom gelben Fleck in der Netzhaut. Zwei Tage später bringt im physikalischen Institut der grosse Magnet, unter Schmerz, den Fremdkörper hinter die Iris, am inneren-oberen Rand. Am folgenden Tage Hypopyon. Narkose, Iridectomie, Entfernung des Splitters $(17 \mathrm{mg}) \mathrm{mit}$ dom kleinen Magneten. Sehschärfe $={ }^{1}, 6$. Umschriebene Linsentrubung.

Fall 7. Ein 23jähriger kam Tags nach der Verletzung mit $3 \mathrm{~mm}$ lanoer Wunde der Lederhaut und einem unten in der Netzhant sitzenden Splitter. Der grosse Magnet im physikalischen Institut bewirkt Schmerz und zieht den Fremdkörper nach vorn, aber unterhalb der Einschlagsstelle. Verband. Der Kranke wird "nach der Klinik verbracht" und chloroformirt, ein kleiner Stich durch die Augenhäute angelegt und der Splitter leicht ausgezogen. Sehschärfe $=1 / 8$.

Verfasser schliesst, dass man mit dem grossen Magneten nicht bloss lose im Glaskörper liegende Eisensplitter ausziehen kann, sondern auch solche, welche fest in der Netzhaut stecken, sofern sie dort noch nieht eingeheilt sind.

Fall 5 war sehr leicht, sowie er kam, mit dem kleinen Magneten durch blosse Wundlüftung zu operiren. Der bleibende Erfolg wäre vielleicht besser gewesen, wenn der grosse Splitter nicht so lange im Glaskörper blieb. Fall 6 lasse ich als Bereicherung unseres Könnens gelten, obwohl er, ebenso gut wie meine oben beschriebenen Fälle 1 und 2 , nach genauer Berechnung des Sitzes

3) Beitr. z. Augenheilk., hrg. von Prof. Deutschmann, Heft XIII, S. 20 fgd., 1894 
mittels des kleinen Magneten operirt werden konnte. Von Fall 7 gilt das gleiche. Derselbe zeigt, dass der grosse Magnet dem Splitter eine falsche, zur Ausziehung ungeeignete Flugrichtung geben kann.

C. In demselben Heft beschrieb Prof. Haab einen neuen Elektromagneten zur Entfernung von Eisensplittern. Es ist ein walzenförmiger Kern aus weichem Eisen, $10 \mathrm{~cm}$ dick, $60 \mathrm{~cm}$ lang, $30 \mathrm{~kg}$ schwer, beiderseits in stumpfe, abschraubbare Spitzen ${ }^{1}$ ) endigend.

Darum sind zwei Spulen von $2 \mathrm{~mm}$ dickem Kupferdraht gewickelt, jede von $28 \mathrm{~kg}$ Gewicht. Das Holzgestell ist $105 \mathrm{~cm}$ hoch und wiegt $51 \mathrm{~kg}$. Der Preis beträgt 550 Franken. - Den Gleichstronl liefert eine Dynamomaschine des physikalischen Laboratoriums. (6-8 Amperes mit 50-60 Volt Spannung. ${ }^{2}$.)

Fall 8. Eine 26jährige kam am Tage der Verletzung. Wunde der Lederhaut von $2 \mathrm{~mm}$ Länge, Splitter in der Netzhaut, $3 \mathrm{~mm}$ unterhalb der Mitte zwischen Sehnerv und Grube. Nach sechs Tagen wird die eine Spitze des neuen Elektromagneten der Wunde angelegt, die Kranke zuckt zusammen. Der Augenspiegel zeigt, dass der Splitter unterhalb der Einschlagswunde sich befindet. Es gelingt nicht, trotz mehrerer Versuche, ihn vollends herauszuziehen. Die Kranke wird in der Klinik gelagert, chloroformirt, die Wunde etwas erweitert und mit dem kleinen Elektromagneten der Splitter $(20 \mathrm{mg})$ herausbefördert, wobei kein namhafter Glaskörperverlust entsteht. Sehschärfe $=1 / 4$.

Fall 9. Ein 43 jähriger zeigte drei Tage nach der Verletzung eine Hornhautnarbe von 1,5 mm Länge, Ausschwitzung in der Pupille, Hypopyon. 14 Tage später brachte der grosse Magnet den Splitter unten hinter die Iris, so dass er nach kleinem Einschnitt mit dem kleinen Magneten ohne Irisausschneidung herausbefördert werden konnte. Nach einenı Monat Finger auf $0,75 \mathrm{~m}$.

Fall 10. Ein 19jähriger kam 9 Uhr vornittags, 6 Stunden nach der Verletzung, mit einer 1,5 mn langen Wunde der oberen Hornhaut, Loch der Iris, leichter Linsentrubung; Splitter nicht zu sehen. An demselben Tage, um $1 / 26$ Uhr Nachmittags, wurde der grosse Magnet angewendet und brachte den Splitter unten hinter die Iris. Bei den Versuchen wurde der Kranke vor Schmerz fast ohnmächtig. Entfernung des Splitters mit dem kleinen Magneten. Langwierige Entzündung (Iritis serosa), Einreibungscur und Atropin.

Fall 11. Ein 27jähriger kommt 26 Tage nach der Verletzung mit einer $1,5 \mathrm{~mm}$ langen Narbe der Hornhaut, Kerbe des Pupillenrandes, abgeflachter Linsentrübung. Der grosse Magnet brachte den Splitter hinter die Iris, mit dem kleinen wurde er ausgezogen. Die Linse trat in den Glaskőrper und ist noch nicht aufgelöst. Wenn die Linsenmasse sich senkt, ist Sehschärfe $=1 / 8$

D. Der zweite, welcher mit Wärme sich des neuen Verfahrens annahm, ist Herr College Schlösser ${ }^{3}$ ).

Fall 12. Der Splitter war durch die "nasale Hornhautnitte" eingedrungen und steckte im Glaskörper nahe bei der Macula. Am Abend desselben Tages wurde der grosse Magnet des Münchener Polytechnicums angewendef. Der Kranke empfand ziemlichen Schmerz, mit dem Augenspiegel war der Splitter nicht mehr zu sehen; aber im nasalen Kammerwinkel erschien frisches Blut. Die temporale Seite des Hornhautrandes wurde an den Magneten gelegt und so der Splitter quer durch die Linse hindurch in die Vorderkammer und aus der Hornhautwunde herausgezogen. Es folgte schleichende Cyclitis und nach sechs Wochen Enucleation.

Fall 13. Ein Splitter, der seit sieben Wochen in der Linse steckte, wurde in die Vorderkammer befördert und am folgenden Tage durch Hornhautschnitt ausgezogen.

Schlösser verfertigte einen Magneten ${ }^{4}$ ) mit kegelförmigem Polschuh. Derselbe erfordert eine Stromspannung von 27-30 Volt und kann an jeder (?) Beleuchtungsanlage statt einer Bogenlampe eingeschaltet werden. Das Auge soll der Polspitze so anliegen, dass die verlängert gedachte Magnetspitze durch die Berührungsstelle nach dem Sitz des Fremdkörpers hingeht.

Ein Splitter unter $10 \mathrm{mg}$ Gewicht wird nicht mehr sicher gezogen, Splitter unter $10 \mathrm{mg}$ dürften kaum in die Tiefe des Auges eindringen.

Die zweite Hälfte des Satzes ist unrichtig. Vgl. II, Fall 9 $(8 \mathrm{mg})$; Fall 10 (8 mg); Fall 11 (9 mg); Fall 22 (41/2 mg); Fall 25 $(6 \mathrm{mg})$; Fall $28\left(3^{1} / 2 \mathrm{mg}\right)$; ferner von meinen vorher angeführten neuen Beobachtungen Fall 2 und $3\left(3^{1}{ }_{2} \mathrm{mg}\right)$.

Es sollte $2 \mathrm{mg}$ heissen, wie auch aus den richtigen Angaben von E. Asmus folgt ${ }^{5}$ ).

1) „Die Spitze muss für solche Fälle wie 7 und 8 etwas in die Wunde "eingesenkt werden können; sie muss also recht spitz sein." $\mathrm{Haab}$, a. a. O., S. 74 .

2) Von Wichtigkeit sind die Versuche des Herrn Prof. Kleiner, welche folgendes lehren: Bei doppelter Stromstärke ist die anziehende Kraft das Vierfache. Bei dreifachem Abstand kann dieselbe Stromstärke nur ein Siebentel der anziehenden Kraft bewirken.

3) Bericht über die Heidelb. Ophth. G. 1893, S. 153

4) Für 100 Mark von Edelmann, Nymphenburgstr. 28, München, zu beziehen.

5) Arch. f. 0., 40. Bd. 1894, I, S. 321.
E. Fall 15. Ein 19 jähriger kam am 15. October 1893 in die Petersburger Augenheilanstalt (Dr. v. Schröd er) mit Hornhautwunde, Linsentrübung, Hypopyon. Am 19. wurde der mit dem inzwischen beschafften Accumulator verbundene Inductionsmagnet auf die Hornhaut aufgesetzt, worauf der grosse Eisensplitter in die Vorderkammer drang. Schnitt mit dem Schmalmesser, Ausziehung des Splitters mit dem kleinen Magneten. (Ausgang unbekannt.)

F. In der neuesten Auflage des Lelirbuches der Augenheilkunde von Prof. Schmidt-Rimpler ${ }^{1}$ ), das überhaupt durch sorgsame Behandlung dieser praktisch so wichtigen Dinge sich vortheilhaft auszeichnet vor anderen, aus denen man alles mögliche lernen kann, nur nicht das Heilen, finden wir die folgende Bemerkung:

"Noch erheblich einfacher ist die vou Haab und Schlösser gerühmte Methode, einfach durch Anlegen eines sehr starken Elektromagneten auf die Eintrittsstelle, das Eisen aus dem Auge herauszubefördern. Leider gelingt auch dies nicht immer; trotz der Benutzung des ungewöhnlich starken Elektromagneten des Göttinger physikalischen Instituts versagte es mir bisher in fünf Fällen viermal: einmal kam das Eisen gleichzeitig mit einem Irisfetzen durch die Hornhautwunde."

Prof. Schmidt-Rinpler gedachte seiner Erfahrungen in einer Verhandlung auf dem diesjährigen internationalen medicinischen Congress zu Rom, worüber leider noch kein zuverlässiger Bericht vorliegt, und war so liebenswürdig, auf meine Bitte mir seinen letzterwähnten Fall für diese Veröffentlichung zur Verfügung zu stellen, wofür ich nicht verfehle, ihm meinen besten Dank auszllsprechen:

Fall 16. „Dem Schlosser Meyer war vor 24 Stunden beim Schmieden ein Stïckchen Eisen in das rechte Auge geflogen. Jetzt (16. November 1893) lebhafte pericorneale Injection, Schmerzen, Gegend des Corpus ciliare in der unteren Hälfte ausgesprochen druckempfindlich. In der unteren Cornealhälfte eine perforirende Wunde, an welcher die Iris zum Theil liegt, Pupillarrand daselbst tief eingerissen. Kleines Hypopyon. Linse getrübt, vom Fremdkörper nichts zll sehen. Handbewegung in $15 \mathrm{~cm}$; für mittlere Lampe Gesichtsfelddefect nach oben. Wir brachten den Patienten in das physikalische Institut. Der durch Maschine betriebene Elektromagnet hatte auf seinem cylinderförmigen Eisenkern (ca. $16 \mathrm{~cm}$ Durchmesser) ein horizontal liegendes, viereckiges Eisen aufliegen, an dessen vorderer Seite $(4 \mathrm{~cm}$ Seitenlänge) ein $1 \mathrm{~cm}$ im Durchmesser haltender, vorn zugespitzter Eisencylinder eingelassen war. An diesen wurde das cocainisirte Auge mit der leicht klaffenden Wunde gebracht. Nach einigen vergeblichen Versuchen fuhr der Verletzte plotzlich vor Schmerz zurück, und an der abgestumpften Spitze hing das Eisenstückchen, etwa $4 \mathrm{~mm}$ lang und $2 \mathrm{~mm}$ breit, sehr dünn. An ihm haftete ein herausgerissenes Irisstück und etwas schleimig graue Masse. Die vordere Kammer hatte sich sofort mit Blut gefullt. Unter antiseptischem Verband resorbirte sich anfinglich das Blut, aussen und oben wurde etwas Iris sichtbar. In der Pupille etwas gequollene Linse. Amaurosis. Später (4. December) wird das Corpus ciliare schmerzhaft, in der vorderen Kammer neue Blutungen und Verkleinerung der Cornea. 7. December: Enucleatio bulbi wegen zu fürchtender sympathischer Ophthalmie.

So sehr uns in obigen Falle die Herausbeförderung des unsichtbaren Eisenstïckchens imponirte, zeigt doch das Herausreissen der Iris und der weitere Verlauf, dass diese Art der Anwendung des Magneten durch secundäre Verletzungen, welche wir nicht voraussehen und kaum vermeiden können, eine gewisse Gefahr in sich schliesst. Es dürfte daher in jedem Einzelfalle zu überlegen sein, ob die äussere Anwendung des Magneten oder das bisher übliche Eingelien nnit demselben vortheilhafter erscheint. Das war die Anschauung, der ich in Rom Ausdrnck geben wollte."

In folgendem Falle gelang die Entfernung mit eingeführtem Magneten, nachdem der grosse Magnet vorher erfolglos angewendet war.

Fall 17. Der Kesselschmied Bode hatte am 5. September 1892 durch Hineinfliegen eines Stückchen Eisenblechs eine Verletzung des, rechten Auges erhalten. Wir fanden am folgenden Tage eine lineare ca. $3 \mathrm{~mm}$ lange Hornhautwunde aussen-unten, in welcher die anliegende Iris lag. Vorderkammer eng, Linse getrübt und gequollen. Pupille relativ weit. Kleinste Lampe, freies Gesichtsfeld. Vom Fremdkörper nichts zu sehen. 8. September vergeblicher Versuch im physikalischen Institut mit dem grossen Elektromagneten, das vermuthete Eisenstückchen zu entfernen. 9. September Iridectomie, Herauslassen der gequollenen Linsenmassen und Eingehen mit dem Hirschberg'schen Magneten in den Glaskolper. Nach einigem Bemühen wurde ein kleines dünnes Eisenplättchen, etwa $1^{1 / 2} \mathrm{~mm}$ im Quadrat, extrahirt. Heilungen mit Schwankungen in der Tension und den Entzündungserscheinungen, Nachstar. 29. November Entlassung. Auge entzündungsfrei. Trübung in den untersten Hornhautpartieen. Ausgedehnter Nachstar; beim Blick nach oben erhält man mit Augenspiegel rothes Licht. Tension etwas geringer als links. Mit +12 D. Sehschärfe $=1 / 12$, Gesichtsfeld frei. “

G. Fall 18. Zu Prof. Deutschmann ${ }^{2}$ ) kam ein 25jähriger, dem seit zehn Tagen hart am Hornhautrande in den Häuten ein Splitter steckte. Derselbe versank beim Versuch mit Hirschberg's Magnet in die Tiefe. Iridectomie und nochmaliges Eingehen vergeblich. Der nach vier Tagen

1) Berlin 1894, S. 310 .

2) Beitr. z. Augenheilk. 1894, 13. Heft, S: 97. 
beschaffte grosse Magnet beförderte den Splitter an die Wunde, der kleine heraus. Sehschärfe $=1 / 4$, leichte Linsenkapselnarbe am Aequator.

Ueber diesen Fall brauche ich keine Kritik zu üben; fügt doch der Herr Verfasser seiner Mittheilung die folgende Bemerkung hinzu: „Solche (Lisensplitter) aber, die in's Innere des Bulbus eingedrungen waren, bei denen es unmöglich war, mit einiger Sicherheit ihre Lage zu diagnosticiren, hatte ich nie das Glück weder mit Instrumenten noch mit Hilfe des Magneten entfernen zu können."

H. Fall 19. Zu Prof. O. Schirmer kommt ein 19 jährigel unmittelbar nach der Verletzung, mit kleiner Wunde der Hormhaut, Loch der Iris, ganz unbedeutender, zarter Trübung der Linse. Der Fremdkörper wird nicht gesehen. Nach zwei Tagen wird er in Glaskörper gefunden, aber nicht ausgezogen. N ach drei Monaten ist die Sehkraft fast aufgehoben. Das Auge wird an deu stärksten Magneten des physikalischen Instituts gelegt, siebenmal für je $1 / 2$ Minute. Heftigster Schnerz erfolgt, der Fremdlkörper bleibt an seiner Stelle, wie mit dem Augenspiegel nachweisbar: Am Nachmittag erfolgt heftigster Glaucom-Anfall. Am nächsten Morgen wird der sehr kleine Splitter nach der "alten Methode“ (Lederhautschnitt und Eilfuhhrung des kleinen Magneten) leicht ausgezogen. Sehschärfe $=1 / 3$, gutes Gesichtsfeld.

Auch diesem Fall brauche ich eine Kritik nicht beizufügen: ich verweise nur auf die Regeln, die ich für mein und die $\mathrm{Haab}$ für sein Verfahren aufgestellt hat.

Schlusswort. Die ungerechtfertigten Vorwïrfe, die gegen das von mir empfohlene Verfahren erhoben sind, glaube ich widerlegt zu haben. Einzelnen Lobpreisern der neuen Belıandlungsart fehlt es an eigner Lrfahrung, mehreren an Berücksichtigung der Literatur und der genauen, aus der Erfahrung gewonnenen Regeln. Ist doch sogar der Satz, dass man mit dem Glaskörper alles machen könne, wenn man nur den Carbolspray anwende, mir zugeschrieben worden, wälırend ich derjenige war, der iln stets auf das kräftigste bekämpfte! $\left.{ }^{1}\right)$

Die Mehrzahl aller Fälle, bei denen man vernünftiger Weise Erfolg erwarten kann, lassen sich mit einem g'uten kleinen Elektromagnet (von 200_500 g Tragkraft, je nach der Dicke des Endstïcks), nach sorgfältigel Diagnose und Abmessung, unter vorsichtigem, sauberem Vorgehen ganz gut erledigen. Für die Mehrzahl der Aerzte wird er auch aus äusseren Gründen vorläufig das einzige Instrument bleiben. Diejenigen, welche einen grossen Magneten sich zulegen können, müssen derartige, dem Verfahren als solchem zuzuschreibende Unglücksfälle, wie in Fall 12, 16, 19, nach Möglichkeit zu vermeiden suchen. Nach längerer Zeit werden sie anch den Vergleich zwischen den beiden Verfahren ziehen können; den kleinen Magneten können sie gar nicht entbehren, sei es zum Schlussact, sei es überhaupt in einer nicht unbeträchtlichen Zahl von Fällen.

Jedenfalls ist es hart und selbst schädlich, die Kranken Tage lang warten zu lassen, bis der grosse Magnet fertig; sie in physikalische Kabinette und zurtick zu schleppen, um daheim die Operation doch erst zu beendigen; sehr unangenehm ist auch der heftige Schmerz, bis zur Ohnmacht, den der grosse Magnet macht, da der Kranke dabei sitzen muss, also nicht betäubt werden kann. Ich selber hatte mir schon seit Jahren die Frage vorgelegt, ob grössere Maschinen oder der Auschluss an die mächtige Leitung unserer Elektrizitatswerke nützlich sei. (Vgl. I, S. 12; II, c. II).

Für die Mehrzahl der Operationen werde ich bei meinem bewährten Instrumente bleiben, das ich kenne, das ich beherrsche und das einen weit grösseren Wirkungskreis besitzt: es holt Splitter tiber und unter $10 \mathrm{mg}$ aus dem Glaskörper, aus der Netzhaut ${ }^{2}$ ), ob sie frisch haften oder schon lange drin stecken, feste und mürbe, ja haarfeine, selbst solche, die zwischen Lederhaut und Strahlenkörper fest eingekeilt waren.

Ls ist ein thatsächlicher Irrthum, dass man immer oder meistens so gewaltiger Magnetkräfte bedürfe, die zu zügeln uns doch recht schwer fällt. In die Wunde möchte auch $\mathrm{Haab}$ seine Spitze gern einführen. Nun man bringe, in frischen Fällen, das $4 \mathrm{~mm}$ breite, $2 \mathrm{~mm}$ dicke Ende meines $500 \mathrm{~g}$ tragenden Magneten sorgsam $\mathrm{z}$ wischen die Lefzen der (nöthigenfalls um 2-3 mm el'weiterten) Hornhautwunde: und hervorschiesst aus der dunklen Tiefe durch den Wundcanal der Linse das Eisensplitterchen, heran an den Magneten, ohne Schmerz und ohne Nebenverletzung und ohne sich abzustreifen, ohne eine falsche Richtung einzuschlagen.

Fall 6. Der 30jährige Hufschmied L. S. schlug mit dem Lisenhammer auf einen Hufeisengriff am 25. Mai 1894, Nachmittags um 1 Uhr: sogleich war die Sehkraft des rechten Auges fort. Nach zwei Stunden, um $3 \mathrm{Uhr}$, erschien er in meiner Anstalt, und nach zehn Minuten war er von seinem Eisensplitter befreit.

Das Auge ist reizlos, in der Mitte der Hornhaut eine leicht verharschte, fast senkrechte Wunde von etwa $2^{1 / 2} \mathrm{~mm}$ Länge, Pupille mittel-

1) Vgl. meine Mittheilung über Glaskörperoperationen, Berlin. klin. Wochenschr. 1885. Ferner Cysticercus in Eulenburg's Realencycl. II. Aufl. u. a. a. 0 .

$\left.{ }^{2}\right)$ Diese ragen wohl oft genug in die Lederhaut hinein. weit (durch vorher genachte Atropineinträuflung), Linse vollständig getribt, Fremdkörper nicht sichtbar. Cocaineintrauflung, Hornhautwunde auf $4-5 \mathrm{~mm}$ enweitert und das $4 \mathrm{~mm}$ breite $(500 \mathrm{~g}$ tragende) Ende meines Magneten zwischen die Wundlefzen gebracht: sofort schiesst, durch den Wundkanal der Linse, der Splitter gegen das Magnetende und wird augenblicklich herausbeforrdert. Verband.

Der Splitter ist blank, $3 \mathrm{~mm}$ lang, $1^{1}{ }_{2} \mathrm{~mm}$ breit, nicht sehr dick und wiegt $10 \mathrm{mg}$. Gerade in diesem Falle, wo der Splitter v om Hufe eines Pferdes stammte, war die rasche Entfernung gewiss recht wünschenswerth. Aber der chirurgische Triumph war doch kein blei. bender Sieg. Zweistündiges Verweilen des septischen Splitters in Glaskörper hatte gonïgt, einen Glaskörperabscess hervorzurufel, del die Entfelnung des Augapfels nöthig machte!

Aber für eine Art von Fällen sind mir die merkwürdigen Versuche von Prof. Haab wichtig und brauchbar; das sind diejenigen, die ich bisher nicht zu operiren pflegte: sie zeigen eine kleine $W$ unde in der Hornhaut oder nahe ihrem Rande, nicht geeignet zur Einführung der magnetischen Sonde, einen kleinen Fremdkörper in der Tiefe, gutes oder befriedigendes Aussehen des Auges und Sehkraft; dazu kommen noch die äusseren Schwierigkeiten der Operation, die in der Individualität und den Verhältnissen liegen. Unter diesen Umständen fühlt der gewissenhafte Arzt immer wieder zum Zuwarten sich veranlasst; inmer wieder erlebt er aber von Zeit zu Zeit, dass solch' ein Verletzter, nachdem er unter Chloralhydrat g'ut geschlafen, am nächsten Morgen die Zeichen des beginnenden Glaskörperabscesses darbietet. Dann wird operirt, der Splitter glänzend geholt, aber für Lrhaltung von Sehkraft ist es zu spät, selbst der Augapfel kann dem Arbeiter nicht immer erhalten werden. Füı diese Fälle scheint mir deı grosse Magnet am Platz, aber von anderer Form, so dass er doch gehandhabt werden kann. So wird die Zahl der Verluste nach diesen schweren und traurigen Verletzungen weiter verringert, der Wirkungskreis des Magneten ausgedehnt werden.

Das wichtigste bleibt allerdings die Verh litung: könnten die Arbeiter dazu gebracht werden, beim Meisseln und Hämmern von Eisen, auch wenn sie nur dabei stehen, ordentliche Schutzbrillen zu tragen, so würde alljährlich eine grosse Zahl von Augen gerettet, eine bedeutende Einbusse von Arbeitsfïhigkeit vermieden werden. 